\title{
Intramedullary Fixation of the Jones Fracture: A case report
}

\author{
by $\mathrm{Al}$ Kline, $\mathrm{DPM}^{1}$
}

The Foot and Ankle Online Journal 2 (6): 2

Intramedullary fixation of the Jones Fracture is described in this case report. Advantages of this procedure include percutaneous fixation of a Jones fracture without the need for traditional open reduction and a more rapid return to weightbearing and activity. The procedure is not technically difficult and provides excellent compression strength. This technique is indicated in stress fracture and/or fractures of a single cortex involving the fifth metatarsal.

Keywords: Intramedullary screw fixation, Jones fracture. reproduction in any medium, provided the original work is properly cited. @The Foot and Ankle Online Journal (www.faoj.org)

I ntramedullary fixation of the fifth metatarsal fracture or Jones fracture is not a new concept. This technique was first described by Kavanaugh, et al., in 1978. Initial techniques employed both the curved Leinbach screw and AO malleolar screw. Unfortunately, these stainless steel screws could break leading to incidence of failure. The procedure subsequently fell out of favor due to this complication. However, with the advent of stronger metals, this technique is once again introduced as a viable alternative to open, plate fixation of the Jones fracture. In April, 2007, the Carolina ${ }^{\text {TM }}$ Jonesfracture system for foot and ankle surgery was launched by Wright Medical Group, Inc. Surgeons at Duke University and OrthoCarolina devised a system that uses high-strength screws specifically designed to treat the Jones fracture using intramedullary compression.

Address correspondence to: Al Kline, DPM

3130 South Alameda, Corpus Christi, Texas 78404.

Email: $\underline{\text { al@kline.net }}$

\footnotetext{
${ }^{1}$ Adjunct Clinical Faculty, Barry University School of Podiatric Medicine. Private practice, Chief of Podiatry, Doctors Regional Medical Center. Corpus Christi, Texas, 78411.
}

There are over 80,000 Jones fractures sustained annually in the United States. ${ }^{1}$ It is now commonly reported that this fracture is under treated and sustains a high non-union rate. Some have reported up to $50 \%$ non-union rate. ${ }^{2}$ Other reports have suggested a delayed-union rate as high as $66 \% .^{3}$ The technique of intramedullary fixation is indicated for stress fracture of the fifth metatarsal with single cortex involvement and without comminution.

The advantage of this procedure is its simplicity and the ability to perform the procedure through a small percutaneous incision. This allows stable fixation of the fracture without a large incision and use of traditional plate fixation.

The intramedullary screw is ideal to promote stabilization of the fifth metatarsal fracture and allow the patient to return to activity sooner. Activity and weight bearing can be resumed as soon as 10 days following surgery. 


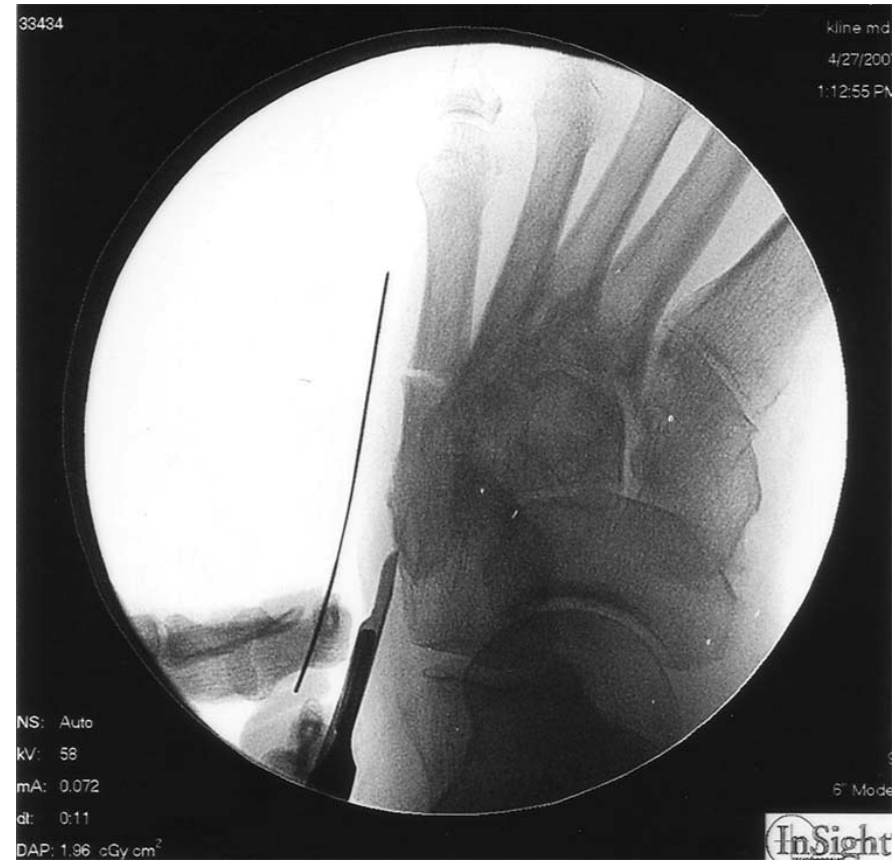

Figure 1 The transverse stress fracture of the fifth metatarsal. A percutaneous incision is made at the base of the fifth metatarsal under fluoroscan.

\section{Case Report}

A 56 year-old female presents with pain and swelling to the lateral border of the foot. She reports slipping down a set of steps and sustaining a "burning pain" to the foot. She denies "twisting" or inverting the foot during injury. She continued to have pain and swelling following the injury and presented to our office. Radiographs confirmed a transverse stress fracture of the fifth metatarsal. The patient has a history of hypertension and hypercholesterolemia. She is a nonsmoker and active. We scheduled the patient for intramedullary screw fixation. Because the patient was relatively sedentary and not athletic, we opted to fix the fracture with a 4.0 TiMax $^{\mathrm{TM}}$ cannulated lag screw.

\section{Surgical Technique}

The patient was brought to the operating room and under IV sedation, a small skin block was performed along the base of the fifth metatarsal.

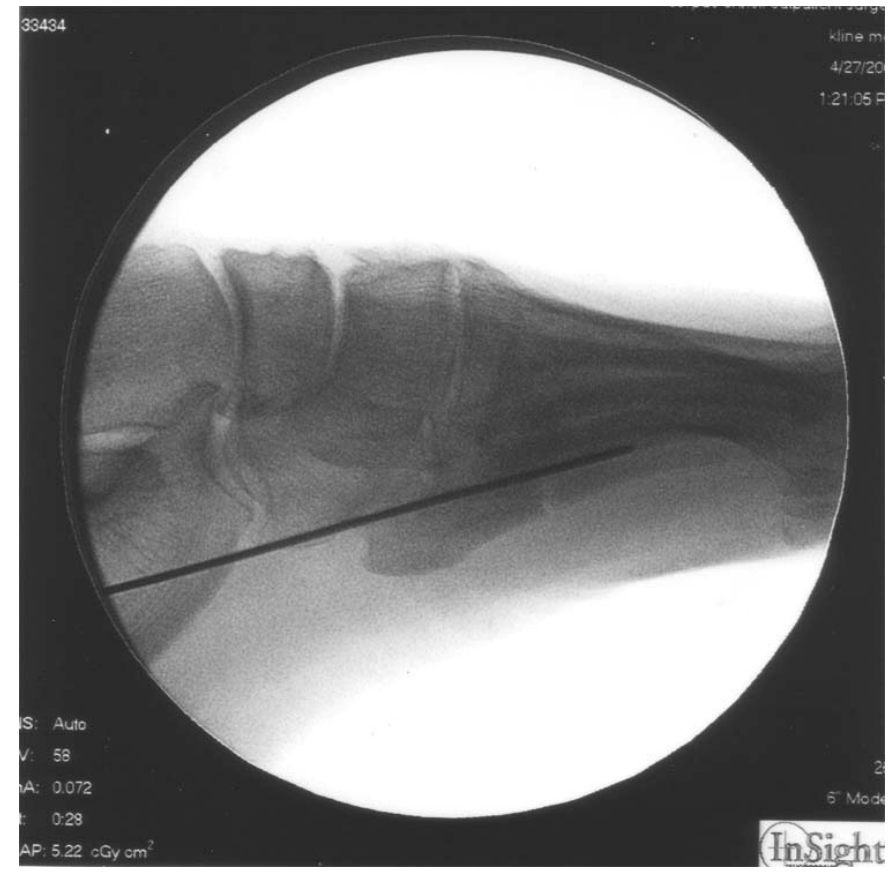

Figure 2 The guide pin is placed across the fracture site to determine proper screw length. Careful counter pressure is maintained at the fifth metatarsal head to prevent further distraction of the fracture site.

Using the Hologic ${ }^{\circledR}$ - InSight Fluoroscan mini C-arm, the fracture is identified. A small, percutaneous incision is made along the base of the fifth metatarsal. A guide wire can be used over the skin under fluoroscan to get a quick idea of the screw length needed for fixation. (Fig. 1).

We used the TiMAX ${ }^{\text {TM }} 4.0$ cannulated lag screw. The lag design allows compression of the fracture site. It is important to place all the threads distal to the fracture site allowing for proximal lag compression when tightening the screw. By placing the run-out of the screw away from the fracture line, this helps to prevent screw fatigue or fracture. A washer is not required. The intramedullary guide wire is then placed across the fracture site and the proper length is determined. (Fig. 2)

It is important to place some counter pressure along the long axis of the fifth metatarsal at the metatarsal head. This will prevent further distraction of the fracture site while drilling. 


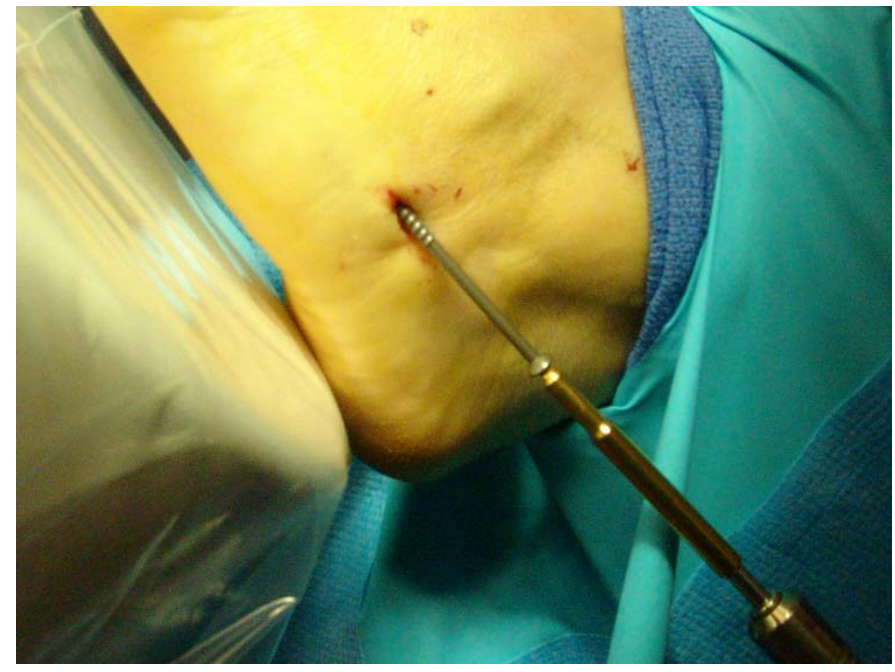

Figure 3 The guide pin is then overdrilled and the screw is placed into the medullary canal. The screw is tightened with compression of the transverse fracture site.

This is also important if the pilot hole is over-drilled. Soft tissue and tendon attachment is protected with a small drill sleeve and then the proper length screw is inserted. (Fig. 3).

As the screw is tightened, you may place counter pressure along the long axis of the fifth ray. Using the fluoroscan, a noticeable 'pinching' of the fracture site will occur due to the compression force of the screw. The low-profile screw head may also be 'buried' into the hard, thick cortical portion of the styloid process ensuring to prevent screw head irritation. Once the screw is in place, it will provide significant stability to the fracture site and allow for earlier weight bearing and activity. (Fig. 4).

A single skin suture or 'butterfly' tape is then placed to close the small incision. The patient is then placed in a posterior splint and kept non-weight bearing for approximately 10-14 days. This can be followed by placing the patient in a CAM walking in a brace or simple post-surgical shoe. The screw may remain in the bone at the discretion of the patient and surgeon.

At 10 weeks post-op, the fracture site is well healed and the patient is walking without assistance or pain. (Figs. 5A and B)

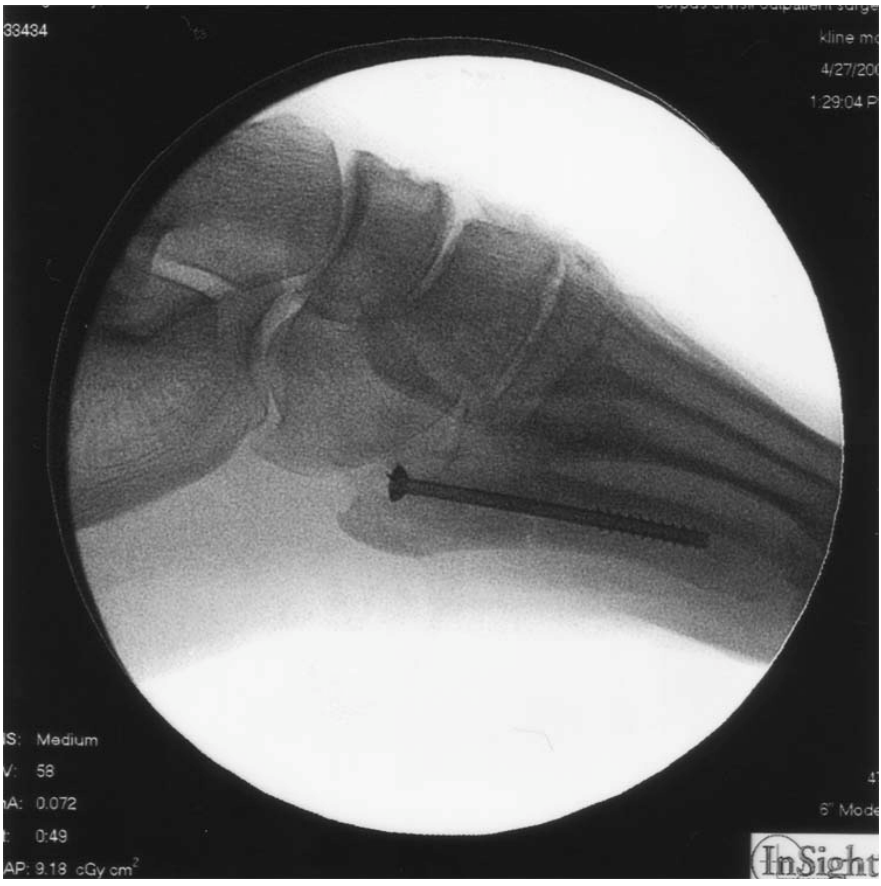

Figure 4 The threads of the lag screw are placed distal to the transverse fracture allowing for proper compression of the fracture. The lag screw provides increased stability of the transverse fracture allowing for an earlier return to weight bearing and activity.

\section{Discussion}

Intramedullary fixation of the Jones fracture is a simple and quick operative procedure. It allows for fracture stability without the need for a large, open reduction procedure. The procedure also allows for quicker weight bearing and return to activity without the risk of refracture.

The mechanism of injury is commonly described as stressed inversion and plantarflexion. In our case, however, the patient slipped and sustained an acute transverse stress fracture of the proximal diaphysis. In Kavanaugh et al., ${ }^{3}$ original article, he also observed simple transverse diaphyseal stress fracture of the fifth metatarsal without a history of stress inversion or plantarflexion. ${ }^{3}$ 


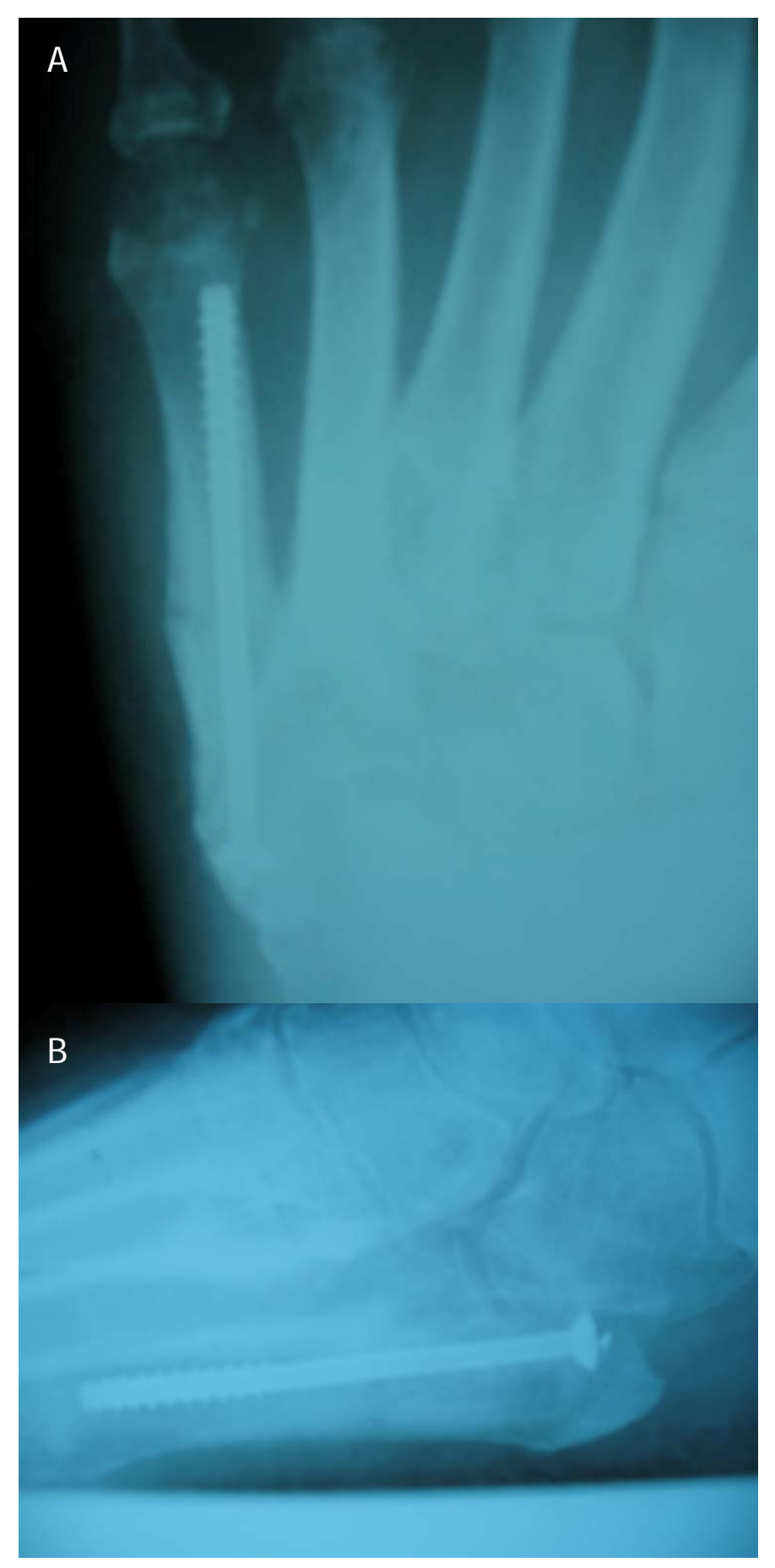

Figure 5A and 5B The fracture site 10 weeks after surgery. The patient has been walking on the fracture site for 8 weeks after being maintained non-weight bearing in a posterior splint for the initial 2 weeks after surgery. The fracture site is stable without signs of refracture or instability.
Using force-platform analysis in eleven of twentythree cases confirmed vertical and mediolateral forces concentrated over the fifth metatarsal causing stress diaphyseal fracture, not inversion stress.

Adduction of the forefoot is thought to potentiate stress leading to stress fracture. He concludes this to be the original Jones fracture as first described by Jones in 1902. He also observes that the stress fracture is difficult to treat and led to delayed union in eighteen of twenty-two patients treated conservatively $(66.7 \%)$. Non-unions and delayed unions continue to be reported in cases of casting, even a stress fracture, since this study was first initiated. Refracture is also high in under treatment of this injury. Kavanaugh, et al., reported nine of twenty-two patients who sustained refracture after immobilization casting for an average of 23.3 weeks! ${ }^{3}$

Wukich, et al., recently reported a higher non-union and refracture rate with intramedullary screw fixation for the Jones fracture in a Division I athlete. This appeared to be due to using a smaller screw diameter. They propose using a larger diameter screw, up to 6.5 $\mathrm{mm}$ in diameter screw to fixate the Jones fracture in competitive athletes. ${ }^{5}$

The high incidence of non-union, delayed union and refracture justifies the need for earlier surgical intervention. Of course, patient needs and activity will be individualized. Although early weight bearing and activity can be achieved as early as 7 to 10 days after intramedullary stabilization of a Jones fracture, the individual weight and activity of the patient should be considered. In the athlete, this procedure allows for earlier activity and training. We advise a posterior splint, non-weight bearing for 2 weeks, with progression to weight bearing and a surgical shoe or CAM boot for an additional 2 to 4 weeks. The patient may then resume normal activity after 6 weeks. 


\section{References}

1. Orthopedic Technology Review.: Product News: JonesFracture System.

2. Ortiguera CJ, Fischer DA: A review of the current treatment for fracture of the proximal fifth metatarsal first described by Jones. Orthopedic Technology Review 2 (4): 2000.

3. Kavanaugh JH, Brower D, Mann RV: The Jones Fracture Revisited. J Bone Joint Surg Am 60A: 776 - 782, 1978. [PDF] 4. Kline A: A review of the Jones fracture with simple classification for conservative versus surgical treatment. Podiatry Internet Journal, 1 (1): 10, 2006. [Online]

5. Wukich DK, Rhim B, Dial DM: Failed intramedullary screw fixation of a proximal fifth metatarsal fracture (Jones fracture) in a division I athlete: A case report. The Foot and Ankle Online Journal, 2 (6): 1, 2009. [Online] 